\title{
A computationally efficient reduced order model to generate multi-parameter fluid-thermal databases
}

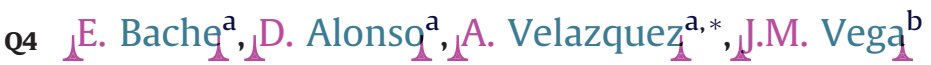 \\ ${ }^{a}$ Aerospace Propulsion and Fluid Mechanics Department, School of Aeronautics, Universidad Politecnica de Madrid, Plaza del Cardenal Cisneros 3, 28040 Madrid, Spain \\ ${ }^{\mathrm{b}}$ Applied Mathematics Department, School of Aeronautics, Universidad Politecnica de Madrid, Plaza del Cardenal Cisneros 3, 28040 Madrid, Spain
}

\section{A R T I C L E I I N F O}

\section{Article history:}

Received 24 April 2011

Received in revised form

31 August 2011

Accepted 31 August 2011

Available online $\mathrm{xxx}$

\section{Keywords:}

Reduced order model

Database generation

Backward-facing step

Non-isothermal flow

Gradient-like minimization methods

\begin{abstract}
A B S T R A C T
A reduced order model (ROM) is proposed to generate multi-parameter databases of some fluid-thermal problems, using a combination of proper orthogonal decomposition, a gradient-like method, and a continuation method. The resulting ROM greatly reduces the CPU time required by slower methods based on genetic algorithm formulations. As a byproduct, the number of required snapshots is also reduced, which yields an additional improvement of the computational efficiency. The work presented in this article aims to facilitate the use of ROMs in industrial environments, in which time is a very important asset. The methodology is illustrated with the non-isothermal flow past a backward-facing step in the laminar regime, which is a representative problem, related to the engineering design of micro-heat sinks.
\end{abstract}

(c) 2011 Elsevier Masson SAS. All rights reserved.

\section{Introduction}

One of the applications of reduced order models (ROMs) in fluid-thermal problems is the non-expensive generation of databases of practical engineering interest. In fact, there are many industrial situations in which the design engineer in charge of a particular problem needs to fill in a multi-parameter database, within a limited time span and, often, with limited resources. In this context, ROMs are potentially attractive candidates to compute the required data because they are much more computationally efficient than their computational fluid dynamics (CFD) counterparts. Obviously, some accuracy is lost in the process but, as is frequent in engineering environments, a balance needs to be reached between accuracy and cost.

Broadly speaking, the mathematical model of a fluid dynamics ROM can be cast either as an interpolation method over a previous sample of CFD solutions (Bui-Thanh [1], Lorente et al. [2], and Amsallem et al. $[3,4]$ ) or using the governing equations to obtain a better approximation. The latter can be classified into two categories: those that reduce the problem to a set of ordinary differential equations (Galerkin projection on a set of previously calculated POD modes [5]) and those that treat the problem as a minimization

\footnotetext{
* Corresponding author. Tel.: +3491336 6351.

E-mail address: angel.velazquez@upm.es (A. Velazquez).
}

1290-0729/\$ - see front matter () 2011 Elsevier Masson SAS. All rights reserved. doi:10.1016/j.ijthermalsci.2011.08.022 problem (searching for the set of modal amplitudes that minimize a pre-defined residual error in the Navier$_{-}$Stokes equations). Examples of the former can be found in, e.g., the articles by Galletti et al. [6], Sirisup and Karniadakis [7], Burkhard et al. [8], Barone et al. [9], Kalashnikova et al. [10] and Rapun and Vega [11], and examples of the latter in the articles by LeGresley and Alonso [12], Alonso et al. [13], and Rouizi et al. [14], and Bache et al. [15]. In both cases, the CPU time needed to compute the ROM is much smaller than the CPU time required to run the CFD solver. As is to be expected, both approaches have their own advantages and disadvantages: Galerkin based ROMs tend to be fast but they tend to require the implementation of some additional artificial stabilization terms in order to converge to a solution, especially in the case of non-linear equations. Curing this instability has deserved great attention in the literature; see, e.g., Kalashnikova et al. [10] and references there in. ROMs based on minimization instead do not require these additional artificial terms but tend to be slower; this is more so if the minimization strategy is based on stochastic methods of the genetic algorithm type $[13,15,16]$.

Against this background, the main object of this paper is to improve the computational efficiency of the latter class of ROMs. This will be done using a faster minimization strategy based on the combination of a gradient-like method and a continuation method. It must be taken into account that the overall efficiency also depends on the number and the selection of the snapshots in the multi-parameter space needed to generate a sufficiently accurate 
modal basis. In other words, the global efficiency of the method depends on: (a) the ROM itself and (b) the selection of the snapshots. Concerning the latter, it happens that the larger the number of computed snapshots the better the accuracy of the modal basis, and the more expensive the generation of the database. In addition to speeding up calculations, the ROM derived in this paper will also allow for reducing the required number of snapshots, which will be a byproduct of the ROM efficiency. The problem of searching for an optimum distribution of snapshots within the multi-parameter space is related to the so-called sampling problems, which have been recently addressed in the context of POD by Astrid et al. [17], Braconnier et al. [18], and Lorente et al. [19]. In these articles, a sampling methodology is ingrained into the search for a combination of database points that yields the POD manifold that contains the information required to fill in the database within a given accuracy.

For the sake of clarity, the method will be illustrated addressing a specific fluid-thermal problem. In particular, this technical work has been written having in mind the context of engineering design of micro_heat sinks. As it is well-known, these devices are making their way into a number of industrial sectors and, accordingly, they need to be extensively characterized. Specially, this is the case whenever safety is a key factor such as it happens in aeronautics and space. Therefore, undertaking development work aiming to facilitate the extensive characterization of these micro-devices may help to ease their dissemination. The selected test problem is the 2-D non-isothermal flow past a backwards facing step, which can be considered as an idealized situation in which a heat sink microchannel presents a sudden expansion. In this test problem, the flow topology is strongly dependent on the Reynolds number (because of the sudden expansion), and on thermal effects (because of the large variations of water viscosity with temperature).

Regarding the organization of the article, the methodology is presented first. Then the test problem is described, results are Q1 given, and, finally, conclusions are presented.

\section{The POD and residual minimization method}

A brief description of the ROM derivation based on residual minimization is provided first, emphasizing some improvements reported in the literature to increase computational efficiency. Then, the advantages and drawbacks of the genetic algorithms and minimization tools in reduced order modeling are discussed. Finally, the combination of gradient-like and continuation methods that is proposed in this paper is developed.

\section{1. $P O D$ and residual definition}

Let us consider a set of $m$ partial differential equations and $n$ boundary conditions, involving $m$ state variables

$E Q_{j}\left(q_{1}, \ldots, q_{m}\right)=0$ in $\Omega, B C_{k}\left(q_{1}, \ldots, q_{m}\right)=0$ at $\partial \Omega$,

for $j=1, \ldots, m$ and $k=1, \ldots, n$, where $\partial \Omega$ is the boundary of the computational domain $\Omega$, and both the equations and boundary conditions may depend on various parameters. These are solved for $N_{0}$ parameter values that must be representative of the parameter range we intend to cover, obtaining $N_{0}$ solutions $q_{j 1, \ldots}, q_{j N_{0}}$, which will be called snapshots. The associated POD modes for each state variable, denoted as $Q_{j 1, \ldots,} Q_{j N_{0}}$, are calculated [5] as the eigenvectors of the associated covariance matrix $R^{j}$, defined as $R_{k l}^{j}=\left\langle q_{j k}, q_{j l}\right\rangle$, where $\langle$,$\rangle is an appropriately defined inner product.$ The square root of the eigenvalues of $R^{j}, \sigma_{j 1, \ldots .} \sigma_{j N_{0}}$, are known as singular values. The state variables are expanded in terms of the POD modes as

$$
q_{j}=\sum_{k=1}^{N_{j}} A_{j k} Q_{j k} .
$$

The singular values are sorted in decreasing order, and the modes are ordered accordingly. The number of retained modes in each state variable, $N_{j}$, is selected such that the truncation error is smaller than some $\varepsilon$, namely

$$
\text { |error } \mid=\sqrt{\sum_{k=N_{j}+1}^{N_{0}}\left(\sigma_{j k}\right)^{2} / \sum_{k=1}^{N_{0}}\left(\sigma_{j k}\right)^{2}} \leq \varepsilon .
$$

The mode amplitudes are calculated minimizing a residual of the equations and boundary conditions. In principle, such residual, $R$, can be defined substituting (2) into the left hand sides of the equations and boundary conditions, squaring, and adding for all points in the computational domain $N_{E}$ and all points in the boundary $N_{B C}$, as

$R=\frac{1}{N_{E}} \sum_{j=1}^{m} \sum_{k=1}^{N_{E}}\left|E Q_{j}\left(x_{k}, y_{k}\right)\right|^{2}+\frac{1}{N_{B C}} \sum_{j=1}^{n} \sum_{k=1}^{N_{B C}}\left|B C_{j}\left(x_{k}, y_{k}\right)\right|^{2}$,

where $E Q_{j}\left(x_{k}, y_{k}\right)$ and $B C_{j}\left(x_{k}, y_{k}\right)$ denote the result of calculating the expansions (2) at the mesh point $\left(x_{k}, y_{k}\right)$ and substituting into the left hand sides of the equations and boundary conditions (2). Note that the continuity equation and the homogeneous boundary conditions (such as no, slip) need not be imposed in the expansion (2). This is because the linear, homogeneous constraints are automatically satisfied by the snapshots and also by the POD modes (which are linear combination of snapshots). Thus, only the remaining equations and boundary conditions need to be considered in the definition of the residual (4).

It should also be noted that, in principle, the residual (4) is computed using the exact solution of the equations and boundary conditions. These are not to be confused with those that are used by the CFD solver, which in fact generally includes additional terms to help convergence. In fact, adding these additional terms will be convenient to avoid spurious solutions, see Section 4 below.

Calculation of the residual involves a computational effort that scales with $N_{E}$, which is usually quite large. As shown in [16], this CPU effort can be greatly reduced considering a smaller number of mesh points, which scales with the number of retained modes. The latter is usually much smaller than the total number of mesh points in the computational domain. The computational effort also depends on the method that is used to minimize the residual.

\subsection{Minimizing the residual with a genetic algorithm}

In our previous work $[13,15,16]$, we have used a genetic algorithm (GA), which is reviewed briefly in Appendix C, to minimize the residual. The advantage of the GA relies in its robustness since, in principle, it provides the global minimum even if (i) no approximation of the solution is available and/or (ii) the residual exhibits several local minima and/or (iii) the residual is very steep (even discontinuous). The main disadvantage of the method is the relatively large (but still much smaller than its counterpart using CFD) CPU time that can be needed for convergence, which is due to both its slow convergence rate and the need to explore a significant part of the POD manifold. In fact, the GA can only explore a finite, user-defined set of values of the POD-amplitudes. Thus, in order to define that part of the POD manifold that is to be explored, an estimate of the solution is needed in practice. This estimate was obtained in $[13,15,16]$ by means of a combination of POD projection and modal interpolation, which required that the snapshots be spread around the parameter space; for each mode amplitude, the span was defined to range between one half and twice the 
estimated value. In other words, the advantage (i) above is somewhat offset in practice. The POD + GA method was thoroughly studied in [16], attending to various issues related to the definition of the residual, the required number of snapshots, the number of retained modes, and the role of CPU errors. In particular, it was seen that attention must be paid to CFD errors, which affect higher order modes and should be filtered out. Otherwise, if too many modes are retained, the residual minimization can yield spurious solutions. There is, of course, a conflict here since a sufficient amount of modes must be retained to obtain a good approximation.

\subsection{Gradient-like and continuation methods}

Gradient-like methods are much faster than the GA due to their super linear convergence rate. However, the standard Newton method exhibits two main difficulties:

- It requires an accurate calculation of the gradient $\left(g_{i}=\partial_{a i} R\right)$ and the Hessian matrix $\left(G_{i j}=\partial_{a_{i} a_{j}}^{2} R\right)$ of the residual, a process that is quite computationally expensive. This difficulty will be overcome replacing the Newton method by a quasi-Newton method. Specifically, a Broyden method [20] will be used that is described in the Appendix A, at the end of the article.

- Both the Newton and quasi-Newton methods are local methods that generally require a good initial guess. This difficulty will be solved using the continuation method described in Appendix B.

The resulting gradient-like + continuation method will be labeled as GL $+\mathrm{C}$ method below.

\section{The test problem}

The problem of 2-D non-isothermal flow past a backward facing $_{2}$ step in the laminar regime is considered. A sketch of the (nondimensional) geometry and the computational domain is presented in Fig. 1 below. The flow moves from left to right and it separates at the step corner yielding a recirculation region, whose length $L_{R}$ depends strongly on the flow parameters. The walls are adiabatic except for a piece of the lower wall downstream of the step, where a wall temperature is imposed. It is considered that the working fluid is water and that both its viscosity and thermal conductivity depend on temperature.

The conservation equations and boundary conditions of the problem are:

$\nabla \cdot \mathbf{u}=0$

$\mathbf{u} \cdot \nabla \mathbf{u}+\nabla p-\frac{1}{\operatorname{Re}} \nabla \cdot\left[\mu\left(\nabla \mathbf{u}+\nabla \mathbf{u}^{T}\right)\right]=0$

$\mathbf{u} \cdot \nabla T-\frac{1}{\operatorname{RePr}} \nabla \cdot[\kappa \nabla T]=0$

where $\mathbf{u}$ is the velocity vector, whose $x$ and $y$ components are denoted as $u$ and $v$, respectively, $p$ is the pressure, $T$ is the

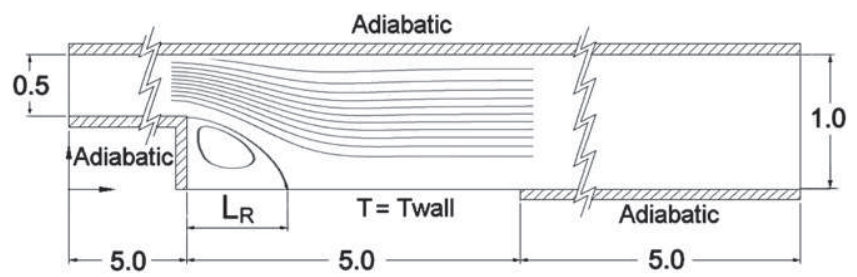

Fig. 1. Sketch of the test problem. temperature, Re is the Reynolds number, Pr is the Prandtl number, $\mu$ is the viscosity, $\kappa$ is the thermal conductivity, $\partial_{x}$ and $\partial_{y}$ stand for partial derivatives and $\Delta=\partial_{x x}^{2}+\partial_{y y}^{2}$ is the Laplacian operator. The spatial variables, the velocity, and the pressure are nondimensionalized with the hydraulic diameter of the inlet section, the inlet average velocity, and the dynamic pressure, $2 \tilde{h}^{\text {inlet }}, \tilde{U}^{\text {inlet' }}$, and $\rho^{\text {inlet }}\left(\tilde{U}^{\text {inlet }}\right)^{2}$, respectively; the non-dimensional temperature is defined as $T=\left(\tilde{T}-\tilde{T}^{\text {inlet }}\right) /\left(\tilde{T}^{\max }-\tilde{T}^{\text {inlet }}\right)$, where tildes denote dimensional quantities and the superscript max stands for the maximum temperature imposed at the non-adiabatic part of the lower wall $(353 \mathrm{~K})$. The working fluid is water, whose viscosity and thermal conductivity are temperature dependent. Assuming that the temperature at the entrance is $\tilde{T}_{\text {inlet }}=293 \mathrm{~K}$ and using wellknown correlations [21] for water, the non-dimensional viscosity and thermal conductivity are given by

$$
\begin{aligned}
& \mu=\mu_{293 \mathrm{~K}}\left(1-1.1292 \times T+0.4904 \times T^{2}\right) \\
& \kappa=\kappa_{293 \mathrm{~K}}\left(1+0.1572 \times T-0.047 \times T^{2}\right)
\end{aligned}
$$

The inlet Reynolds and Prandtl numbers are defined as $\operatorname{Re}=2 \tilde{\rho}_{\text {inlet }} \tilde{h}_{\text {inlet }} \tilde{u}_{\text {inlet }} / \tilde{\mu}\left(\tilde{T}_{\text {inlet }}\right)$ and $\operatorname{Pr}=\tilde{c}_{p} \tilde{\mu}\left(\tilde{T}_{\text {inlet }}\right) / \tilde{\kappa}\left(\tilde{T}_{\text {inlet }}\right)$

Concerning the boundary conditions, at the inlet section, $x=0$, the flow is assumed to be Poiseuille-like and the temperature, equal to the coolant temperature,

$$
\begin{aligned}
& u=-24(y-1)\left(y-\frac{1}{2}\right), \quad v=0, \quad \partial_{x} p=\frac{-48}{\operatorname{Re}}, \\
& T=0 \quad \text { at } x=0
\end{aligned}
$$

and a stress free boundary condition $\left(\partial_{x} u=\partial_{x} v=\partial_{x x}^{2} p=\right.$ $\left.\partial_{x} T=0\right)$ is imposed at the outlet section. The boundary condition at the non-adiabatic part of the lower wall is

$u=v=0, \quad T=T_{\text {wall }}$ if $5<x<10$ and $y=0$.

The remaining part of the lower wall and the upper wall are thermally insulated. Note that only the non homogeneous boundary conditions (11) and (12) have been displayed. As explained in Section 2.1, the homogeneous boundary conditions need not be considered in the construction of the ROM.

Note that we are giving boundary conditions for pressure at both the entrance and the exit of the domain, which in principle are not necessary because the pressure is just a Lagrange multiplier in incompressible the Navier-Stokes equations, which is needed to compensate for the (extra) continuity equation. But the boundary conditions for pressure are just the ones that match with parallel flow at both the entrance and exit. A pressure boundary condition at the entrance is explicitly given (and used in the ROM construction) because this is convenient to improve precision in the analysis of next section.

CFD computations are carried out in an equispaced mesh that exhibits 32,051 points, using the steady-state version of the flow solver developed by one of the authors of the present article, described in [22]. In particular, the solver is based on a pseudocompressibility approach (see [22,23] for details), which also requires a boundary condition for pressure at solid walls. This is obtained integrating (near the wall) the momentum equation in the direction perpendicular to the wall (except at the corners, where the direction that bisects the corners is considered), with one sided (into the flow domain) derivatives, and imposing no slip at the wall. The solver includes various stabilizing terms in all equations, which are included to avoid instabilities without the need of using too fine of a mesh. These stabilizing terms will be considered below. Additional details of the solver are not needed in this article. 


\section{Results}

The GL $+C$ method will be applied to the test problem in the following parameter range (Fig. 2 ): $50 \leq \operatorname{Re} \leq 250$ and $0 \leq T_{\text {wall }} \leq 1$.

Three figures of merit will be considered, namely the horizontal reattachment length, $L_{R}$, defined as the horizontal length on the lower wall of the recirculation bubble (see Fig. 1), the pressure drop $\Delta P$, defined as the difference between the vertically averaged pressure values in the outflow and the inflow sections of the computational domain, and the Nusselt number, defined as in $[13,15,16]$, namely

$N u=\frac{\tilde{Q}^{\prime}}{2\left(\tilde{T}_{\text {max }}-\tilde{T}_{\text {inlet }}\right) \kappa\left(\tilde{T}_{\text {wall }}\right) \tilde{h}_{\text {inlet }}}=\int_{5}^{10} \partial_{y} T(x, 0) d x$

where $\tilde{Q}^{\prime}$ is the (dimensional) heat flux through the non-adiabatic part of the lower wall. The relative error in the figures of merit is defined as the difference between their values computed by the ROM and the CFD solver divided by their respective mean CFDvalues throughout the parameter space, which are $\overline{L_{R}}=1.99$, $\overline{\Delta P}=-2.19$, and $\overline{\mathrm{Nu}}=3.08$, and errors are defined in \%. In other words, errors are defined as

$\operatorname{Error}\left(L_{R}\right)=\frac{\left|L_{R, \mathrm{ROM}}-L_{R, \mathrm{CFD}}\right|}{\bar{L}_{R}} \times 100$

$\operatorname{Error}(\Delta p)=\frac{\left|\Delta p_{\mathrm{ROM}}-\Delta p_{\mathrm{CFD}}\right|}{\overline{\Delta p}} \times 100$

$\operatorname{Error}(\mathrm{Nu})=\frac{\left|\mathrm{Nu}_{\mathrm{ROM}}-\mathrm{Nu}_{\mathrm{CFD}}\right|}{\overline{\mathrm{Nu}}} \times 100$

These errors will be computed at the five test points P01,..,P05, whose location in the parameter space is given in Table 1 , where the specific values of the CFD computed figures of merit are also provided.

To begin with, POD is applied to the 25 CFD calculated snapshots indicated in Fig. 2. These snapshots have proven to be representative enough [16] for the steady states in the considered parameter space.

The number of modes that are to be retained in each flow variable is now decided according the a priori error estimate (3).

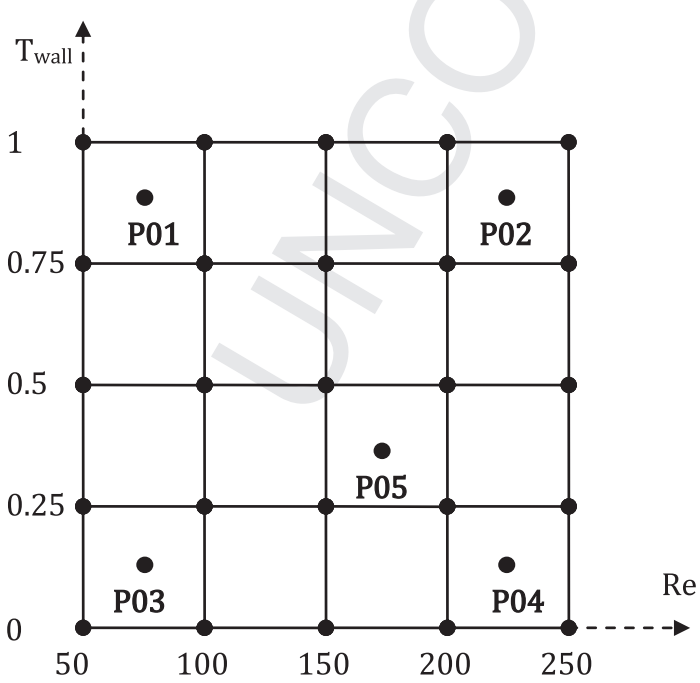

Fig. 2. Parameter space in the $T_{\text {wall }}$ vs. Re plane, snapshots (the 25 cross points of the mesh), and test points (P01-P05).
Table 1

Test points coordinates and figures of merit.

\begin{tabular}{lrllll}
\hline Test point & \multicolumn{1}{c}{ Re } & $T_{\text {wall }}$ & $L_{R}$ & $\Delta P$ & $\mathrm{Nu}$ \\
\hline P01 & 75 & 0.875 & 1.28 & -3.59 & 4.48 \\
P02 & 225 & 0.875 & 2.78 & -0.98 & 6.15 \\
P03 & 75 & 0.125 & 1.18 & -3.71 & 0.57 \\
P04 & 225 & 0.125 & 2.64 & -1.01 & 0.74 \\
P05 & 175 & 0.375 & 2.26 & -1.38 & 2.47 \\
\hline
\end{tabular}

Fig. 3 shows the normalized energy associated with each POD mode, $E_{i}=\sigma_{i}^{2} / \sum_{i} \sigma_{i}^{2}$, vs. the mode number. Note that the number of nonzero singular values in the velocity components and the pressure is equal to the number of snapshots, namely 25; the temperature instead only shows 20 nonzero singular values because the 5 snapshots associated with $T_{\text {wall }}=0$ are strictly isothermal. Also note that the energy decays fairly fast in the four flow variables, which means that the redundancies associated with the parameters are well accounted for by POD. The error bound $\varepsilon=$ $10^{-4}$ in (3) (which approximately corresponds to the energy level $E_{i}=10^{-8}$ in Fig. 3 ) is a good root mean square (RMS) error bound in this problem [16] and yields the following numbers of modes

14, 17, 8, and 19 modes

in the flow variables $u, v, p$, and $T$, respectively. The disparity in the required number of modes is due to the spatial structure of the flow variable distributions. The temperature fields are more complex than the velocity and pressure fields (appreciated in the slower decay in Fig. 3) and require retaining almost all available modes. Going beyond this energy level of the last temperature mode would not improve the description of the temperature field, since almost all modes on the temperature are already retained. The pressure requires fewer modes than the velocity components (see Fig. 3) because it remains almost constant in the $y$-direction in most of the fluid domain.

The truncated expansions resulting from retaining the numbers of modes (16) are substituted into the residual $R$, defined as in eq. (4), with $E Q_{j}$ and $B C_{j}$ standing for the momentum and energy eqs. (6)-(8) and the boundary conditions (11)-(12). Note that (as anticipated in Section 2) the continuity eq. (5) and the remaining boundary conditions are homogeneous and need not be considered. Also, as anticipated right after eq. (4), the residual is calculated using only a limited number of test points. Based on

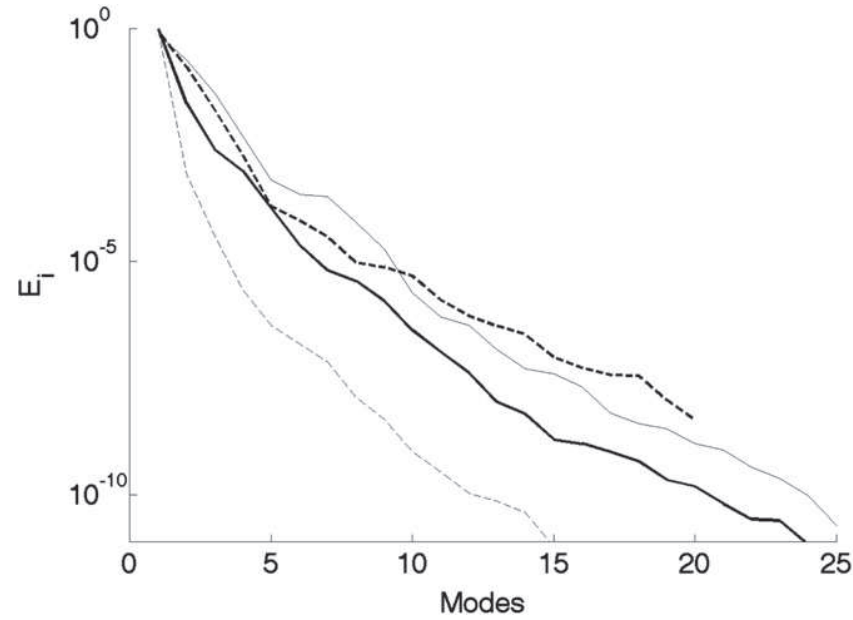

Fig. 3. Normalized energy of the POD modes for $u$ (solid, thick line), $v$ (solid, thin line), $p$ (dashed, thin line), $T$ (dashed, thick line). 
a former calibration in reference [16], the residual is calculated using 84 mesh points uniformly spread out in the following rectangular projection window: $5.5 \leq x \leq 10,0<y<1$.Note that using these 84 mesh points (instead of the 32,051 points contained in the computational mesh) greatly reduces the CPU time needed to calculate the residual. The resulting residual is then used in the $\mathrm{GL}+\mathrm{C}$ method (namely, it is minimized using the gradient-like method described in the Appendix A, which is combined with the continuation method described in Appendix B); at each test point, continuation is performed with $\varepsilon_{1}=10^{-5}$ from the nearest snapshot in the parameter space. For reference, the errors of the results obtained minimizing the residual with a GA (as explained in Section 2.2) are also shown in Table 2. The CPU time required to calculate each flow distribution using the GL $+\mathrm{C}$ method is of the order of $2 \mathrm{~s}$, which compares well with the CPU times required by both the GA (10 min) and the CFD solver (6 h). In addition, construction of the ROM requires running the CFD solver to calculate the snapshots, which is by far the most computationally expensive part of the process. In this context, an effective method to minimize the required number of snapshots would be quite convenient, as already mentioned in Section 1.

Table 2 shows that, unfortunately, the results with the GL $+\mathrm{C}$ method are not as good as could be expected, although it is to be noted that a $10 \%$ error is the typical band discrepancy when performing MEMS experimental testing. In particular, the reattachment length at the test point P04 and the Nusselt number at the test points P01 and P02 show errors that are too large. The results with the GA are closer to their CFD counterparts than those provided by the $\mathrm{GL}+\mathrm{C}$ in some cases (test points 4 and 5) even though the $\mathrm{GL}+\mathrm{C}$ method yields a smaller residual. This is unexpected because the convergence of the $\mathrm{GL}+\mathrm{C}$ method is much better than that of the GA. Thus, a closer look at the GL $+\mathrm{C}$ method is necessary. The possible reasons for the discrepancy are:

1. The retained number of modes is not sufficiently large.

2. The residual is calculated with not enough mesh points.

3. The continuation method is not fine enough.

4. The $\mathrm{GL}+\mathrm{C}$ converged solutions are local minima, and not the global minima that result from the GA.

5. The residual used by the ROM is based on the exact Navier-Skokes + energy equations, while the CFD solver includes some artificial stabilization terms.

These five possible reasons are now considered. The first possibility is checked retaining all modes: 25 modes per variable except $T$, which only has 20 modes because of redundancies (the temperature fields in the five lowest snapshots in Fig. 2 are all identically zero), giving a total number of 95 modes. Results are shown in Table 3. The counterparts using the GA are not provided because of the huge computational time that would be needed with this number of modes; note that the GA CPU time increases exponentially with the number of unknowns, while it increases only

Table 2

Relative errors in \% resulting from minimizing the residual with both the GA and the $\mathrm{GL}+\mathrm{C}$ methods, retaining the numbers of modes displayed in (14). Errors smaller than $0.1 \%$ are rounded off to zero hereafter.

\begin{tabular}{|c|c|c|c|c|c|c|}
\hline \multirow[t]{2}{*}{ Test point } & \multicolumn{3}{|l|}{ GA } & \multicolumn{3}{|c|}{$\mathrm{GL}+\mathrm{C}$} \\
\hline & $L_{R}(\%)$ & $\Delta P(\%)$ & $\mathrm{Nu}(\%)$ & $L_{R}(\%)$ & $\Delta P(\%)$ & $\mathrm{Nu}(\%)$ \\
\hline P01 & 0 & 0.4 & 1.5 & 1 & 1.3 & 8.7 \\
\hline P02 & 0 & 0.2 & 8 & 4 & 0.5 & 7.2 \\
\hline P03 & 0 & 0.9 & 5.2 & 0 & 1.7 & 0.8 \\
\hline P04 & 0 & 0.1 & 5.9 & 9.1 & 0.5 & 3.1 \\
\hline P05 & 0 & 0.2 & 1.6 & 4 & 0.1 & 2.6 \\
\hline
\end{tabular}

Table 3

Relative errors in \% resulting from minimizing the residual with the GL $+\mathrm{C}$ method, retaining all modes ( 25 for $u, v$, and $p ; 20$ for $T$ ).

\begin{tabular}{llll}
\hline Test point & \multicolumn{2}{l}{$\mathrm{GL}+\mathrm{C}$ method } & \\
\cline { 2 - 4 } & $L_{R}(\%)$ & $\Delta P(\%)$ & $\mathrm{Nu}(\%)$ \\
\hline P01 & 1 & 0.6 & 0.7 \\
P02 & 0 & 0 & 9.3 \\
P03 & 0 & 0 & 1.4 \\
P04 & 7 & 0 & 3.3 \\
P05 & 2 & 0.5 & 3.2 \\
\hline
\end{tabular}

slightly when using the GL $+C$ method, which only requires 9.3 CPU seconds per 5 points. Table 3 shows that some of the results are somewhat improved, but the errors in the reattachment length at point P04 and the Nusselt number at P02 are still quite large. Thus, it is concluded that increasing the number of modes does not solve the difficulty.

Similarly, the possible reasons 2 and 3 are checked repeating the calculations using a residual based on all points in the projection window (11,075), and also refining the continuation method. The results (omitted here for the sake of brevity) do not improve, showing that none of these two reasons is responsible for the discrepancy.

In order to further check that none of the first three reasons above is responsible for the discrepancies, we use the method to reconstruct some of the snapshots themselves, taking as initial guess projection of the CFD solution on the POD modes. Retaining all modes, the GL $+C$ method yields the results shown in Table 4 .

This table makes it very clear that something is awry in the GL + C method, which is finding a different solution from that provided by the CFD solver in those snapshots with larger Reynolds numbers, which are the most demanding ones from the computational point of view: the $\mathrm{GL}+\mathrm{C}$ method is finding a spurious solution, fairly different from the CFD solution, in spite of the fact that, since all the modes are retained, the initial guess (projection of the CFD solution onto the POD manifold) exactly coincides with the CFD calculated snapshot. This leaves us with only the last two possibilities anticipated above.

Let us now check the local/global character of the minima of the residual. The converged values of the residuals in Table 2 provided by the GA and the GL $+C$ are of the order of $2 \times 10^{-5}$ and $5 \times 10^{-6}$, respectively. Comparison of the associated $\mathrm{POD}_{7}$ modes amplitudes is also enlightening. The converged amplitudes at these values turn out to be fairly different at the problematic test points. For illustration, the first 10 mode amplitudes provided by the GA associated with the temperature at the test point P02 are $1.01,-1.4 \times 10^{-2}$, $2.3 \times 10^{-3},-1.5 \times 10^{-3}, 3.7 \times 10^{-4},-1.1 \times 10^{-4},-5.6 \times 10^{-5}$, $1.6 \times 10^{-5},-1.2 \times 10^{-5}$, and $-2.7 \times 10^{-6}$, while their counterparts provided by the GL $+\mathrm{C}$ are $1.25,-3.3 \times 10^{-2}, 1.1 \times 10^{-2}$, $4.2 \times 10^{-4},-4.2 \times 10^{-6},-1.7 \times 10^{-3}, 8 \times 10^{-5}, 5.9 \times 10^{-4}$, $7.1 \times 10^{-5}$, and $2.1 \times 10^{-4}$. Thus:

a) It is the amplitudes of the higher order modes that show the largest relative discrepancies. And the values of the higher

Table 4

Relative errors in \% obtained with the GL $+C$ method, retaining all modes ( 25 for $u, v$, and $p ; 20$ for $T$ ) in five snapshots.

\begin{tabular}{llllc}
\hline $\operatorname{Re}$ & $T_{\text {wall }}$ & $L_{R}(\%)$ & $\Delta P(\%)$ & $\mathrm{Nu}(\%)$ \\
\hline 50 & 1 & 0 & 0.3 & 0.9 \\
100 & 1 & 1 & 0.4 & 0.2 \\
150 & 1 & 0 & 0.9 & 5.6 \\
200 & 1 & 1 & 1 & 10.9 \\
250 & 1 & 4 & 0.8 & 12.3 \\
\hline
\end{tabular}


order modes provided by the ROM are generally larger than their GA counterparts.

b) The residual is better minimized by the $\mathrm{GL}+\mathrm{C}$ method, but in spite of this, the solution provided by the GA is closer to its CFD counterpart. This is due to the fact that the spurious solution provided by the $\mathrm{GL}+\mathrm{C}$ method exhibits quite large higher mode amplitudes, which cannot be reached by the GA, since it is outside the span allowed in the GA (see Section 2.2). Limiting the span in the GA somewhat filters spurious solutions, which are allowed in the $\mathrm{GL}+\mathrm{C}$ method. In other words, these spurious solutions (with smaller residuals than the correct solution) were not accessible to the GA (Tables 2 and 3 ) for the simple reason that the span allowed in the GA searching excluded them.

The fact that the spurious solution provided by the $\mathrm{GL}+\mathrm{C}$ method is associated with too large higher order mode amplitudes suggest that CFD errors might play a role in the discrepancies. In other words, the gradient ${ }_{7}$ like method minimizes a residual based on the exact equations, but using modes resulting from CFD calculations (which involve CFD errors not accounted for in the residual calculation) and selecting spurious values of the higher order modes amplitudes.

Two types of CFD errors are present:

i. Standard discretization errors. These could only be avoided using either a finer mesh (which would increase the computational effort to calculate the snapshots) or a better discretization scheme instead of equispaced finite differences (which would make the proposed method strongly dependent on the CFD mesh).

ii. Since the calculation of the residual is based on the exact governing equations, the effect of the artificial stabilizing terms that are added to the CFD solver are seen as errors by the ROM.

If the culprits of the spurious solutions were the errors of the type (i), then the effectiveness of the ROM would be limited. Let us analyze the role of the second type of "errors", which leads us to the fifth possible reason mentioned above. To this end, we replace eqs. (5) - (8) by the steady ${ }_{7}$ state equations that are actually CFD solved. This results in adding to the left hand sides of eqs. (5)-(8) the following four terms

$\delta_{1} \Delta p+\delta_{2} \Delta^{2} p, \quad \delta_{3} \Delta^{2} u, \quad \delta_{4} \Delta^{2} v$, and $\delta_{5} \Delta^{2} T$

which are added to the continuity equation, the horizontal and vertical momentum equations, and the energy equation, respectively, with $\delta_{1}=1.6 \times 10^{-5}, \delta_{2}=2.56 \times 10^{-8}$, and $\delta_{3}=\delta_{4}=\delta_{5}=$ $2.56 \times 10^{-6}$. Using the resulting new equations in the calculation of the residual, the $\mathrm{GL}+\mathrm{C}$ method produces the results shown in Table 5, for the same snapshots considered in Table 4.

The GL $+C$ method now produces acceptable solutions (within $2 \%$ of the CFD solutions) in the five snapshots. Thus, it seems that the reason for the spurious solutions has been found.

Table 5

Counterpart of Table 6 retaining all modes and calculating the residual with the modified governing equations that include the artificial stabilizing terms (mimicking the CFD solver).

\begin{tabular}{lllll}
\hline $\operatorname{Re}$ & $T_{\text {wall }}$ & $L_{R}(\%)$ & $\Delta P(\%)$ & $\mathrm{Nu}(\%)$ \\
\hline 50 & 1 & 1 & 0.1 & 1.7 \\
100 & 1 & 1 & 0.7 & 0.8 \\
150 & 1 & 1 & 1 & 1.1 \\
200 & 1 & 1 & 0.8 & 0.8 \\
250 & 1 & 0 & 0.4 & 1.4 \\
\hline
\end{tabular}

Table 6

Counterpart of Table $5(\mathrm{GL}+\mathrm{C}$, with artificial stabilizing terms) at the five test points, retaining the modes indicated in eq. (14); continuation is initiated at the indicated snapshots.

\begin{tabular}{llllll}
\hline Initial Re & Initial $T_{\text {wall }}$ & Test point & $L_{R}(\%)$ & $\Delta P(\%)$ & $\mathrm{Nu}(\%)$ \\
\hline 50 & 0 & $\mathrm{P} 01$ & 2 & 0.7 & 3.5 \\
250 & 1 & $\mathrm{P} 01$ & 2 & 0.7 & 3.5 \\
50 & 0 & $\mathrm{P} 02$ & 2 & 0 & 0.6 \\
250 & 1 & $\mathrm{P} 02$ & 2 & 0 & 0.6 \\
50 & 0 & $\mathrm{P} 03$ & 1 & 1 & 2.5 \\
250 & 1 & $\mathrm{P} 03$ & 1 & 1 & 2.5 \\
50 & 0 & $\mathrm{P} 04$ & 3 & 0 & 4.2 \\
250 & 1 & $\mathrm{P} 04$ & 3 & 0 & 4.2 \\
50 & 0 & $\mathrm{P} 05$ & 0 & 0.1 & 0.5 \\
250 & 1 & $\mathrm{P} 05$ & 0 & 0.1 & 0.5 \\
\hline
\end{tabular}

Nevertheless, the results in Table 5 are obtained with all modes retained, and must be confirmed using the same number of POD modes retained in the construction of the ROM above. Thus, we use the $\mathrm{GL}+\mathrm{C}$ method with the artificial dissipation terms included, retaining the modes indicated in eq. (14), to calculate the solutions at the five test points. Results are provided in Table 6, as obtained initiating continuations at two of the corners of the parameter space, as indicated in the caption. Note that now the results are reasonably good and, furthermore, they are independent of the point where continuation is initiated. Thus, the difficulty has been solved.

In the Introduction, it was stated that the Galerkin procedure exhibits a higher order mode truncation instability while the present method does not. This remains true as the present method converges to the CFD solution without needing any additional artificial stabilizing terms, not contained in the CFD formulation. In other words, the ROM inherits the instability of the CFD code, but it does not produce any additional intrinsic higher order mode instabilities, as the Galerkin method does. If CFD were based on the exact equations, no additional stabilizing terms would be needed.

Summarizing, the ROM proposed in this article consists in minimizing the residual with the $\mathrm{GL}+\mathrm{C}$ method (Appendices $\mathrm{A}$ and $\mathrm{B})$, calculating the residual with the equations considered by the CFD solver (artificial stabilizing terms included). This method has been checked to be both consistent and robust, which is further illustrated now with two extensions.

A rougher continuation method (with larger continuation steps) produces the same results. In fact, we have checked that one continuation step (which only requires less than two CPU seconds) is enough to compute all test points, with the initial condition located at any of the 25 snapshots. In other words, the GL $+C$ method converges to the right solution when the initial guess is any of the CFD calculated snapshots. This means that continuation is not really necessary for this particular test problem, which must be due to an appropriate convexity property, which could not be guessed a priori. Such a convexity property is not expected in general fluid dynamic-thermal problems, which will generally require using the continuation method.

Table 7

Comparison of the relative errors when using the 25 original snapshots and the 16 snapshots on the boundary on the parameter space.

\begin{tabular}{llllllll}
\hline \multirow{2}{*}{ Test point } & \multicolumn{3}{l}{25 snapshots } & & \multicolumn{3}{l}{16 snapshots } \\
\cline { 2 - 3 } & $L_{R}(\%)$ & $\Delta P(\%)$ & $\mathrm{Nu}(\%)$ & & $L_{R}(\%)$ & $\Delta P(\%)$ & $\mathrm{Nu}(\%)$ \\
\hline P01 & 2 & 0.7 & 3.5 & & 1 & 0.8 & 2.7 \\
P02 & 2 & 0 & 0.8 & & 2 & 0.1 & 0.5 \\
P03 & 1 & 1 & 2.5 & & 1 & 1.1 & 2.1 \\
P04 & 3 & 0 & 4.2 & & 2 & 0.1 & 4.2 \\
P05 & 0 & 0.1 & 0.5 & & 0 & 0.2 & 0.6 \\
\hline
\end{tabular}


Table 8

Counterpart of Table 7 using the 13 snapshots indicated in Fig. 3.

\begin{tabular}{llllllll}
\hline \multirow{2}{*}{ Test point } & \multicolumn{3}{l}{25 snapshots } & & \multicolumn{3}{l}{13 snapshots } \\
\cline { 2 - 3 } & $L_{R}(\%)$ & $\Delta P(\%)$ & $\mathrm{Nu}(\%)$ & & $L_{R}(\%)$ & $\Delta P(\%)$ & $\mathrm{Nu}(\%)$ \\
\hline P01 & 2 & 0.7 & 3.5 & & 0 & 0.6 & 6.0 \\
P02 & 2 & 0 & 0.8 & & 3 & 0 & 1.1 \\
P03 & 1 & 1 & 2.5 & & 1 & 0.8 & 2.6 \\
P04 & 3 & 0 & 4.2 & & 3 & 0 & 4.3 \\
P05 & 0 & 0.1 & 0.5 & & 1 & 0.1 & 0.3 \\
\hline
\end{tabular}

The second extension results from the observation that we have a robust ROM, able to calculate the solution at any point of the parameter space, initiating continuation at any point of the parameter space. Thus, the CFD calculated snapshots are only needed to compute the POD manifold. The latter should require a smaller number of snapshots than those required in reference [16], where an initial guess of the solution was necessary to define the genetic algorithm span. In order to check this, the number of retained snapshots will be decreased, with snapshots located in the parameter space in two different fashions, namely either concentrated in the boundary or spread in the parameter space, emphasizing the robustness of the method. As an example of concentrated snapshots, only those 16 snapshots indicated with crosses in Fig. 3 (located at the boundary of the parameter space) are considered. The resulting POD manifold is truncated as above (with the same RMS error), which now requires $12,13,8$, and 10 modes in $\mathrm{u}, \mathrm{v}, \mathrm{p}$, and $\mathrm{T}$, respectively. The resulting errors in the figures of merit are shown in Table 7, which shows that reducing the number of snapshots from 25 to 16 (which essentially divides by 1.5 the CFD computational effort) produces, basically, the same results.

As an example of more evenly distributed snapshots, the 13 snapshots indicated with black filled diamonds in Fig. 3 are selected. Truncating the associated POD manifold as above requires $12,13,8$, and 11 modes in $\mathrm{u}, \mathrm{v}, \mathrm{p}$, and T, respectively. The associated results are shown in Table 8 , which are almost as good as those obtained above, and show that location of the snapshots in the parameter space is not critical to the solution. The number of modes that need to be retained will change however. Even though the number of snapshots has been decreased by three, the number of modes retained is basically the same as when 16 snapshots were retained. This is not surprising since the 13 snapshots are distributed in a more even fashion and each snapshot contributes less redundant information to the POD basis.

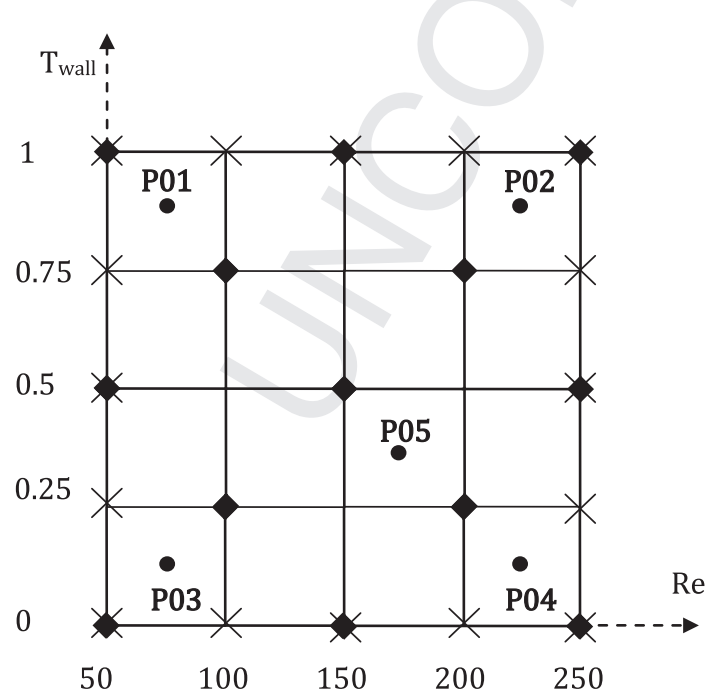

Fig. 4. Location of the two sets of 13 (diamonds) and 16 (crosses) snapshots in the parameter space; location of the test points is also recalled.
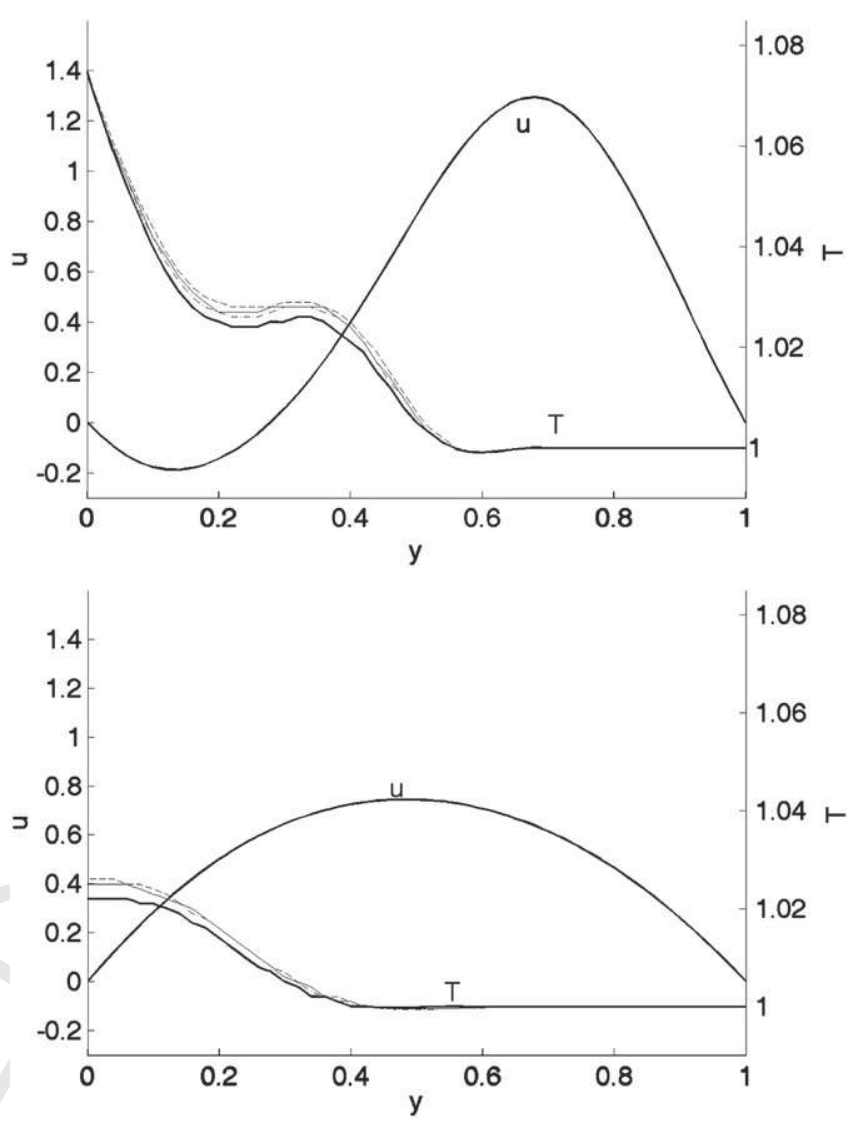

Fig. 5. Distributions of the horizontal velocity and the temperature at the test point P05, at $x=6$ (top) and $x=13$ (bottom) for CFD (solid, thick line), 13 snapshots (solid, thin line), 16 snapshots (dashed line), and 25 snapshots (dash-dotted line).

The results above show that the ROM developed in Section 4 provides the figures of merit with reasonable accuracy. It also approximates well the distributions of the flow variables in the computational domain, as illustrated in Fig. 4, where the vertical distributions of $u$ and $T$ are provided at two representative sections, namely $x=6$ (within the circulation region) and $x=13$ (in the downstream region), as obtained with the three sets of snapshots considered above (Fig. 5).

A ROM has been developed that is based on a combination of POD-mode expansions (from a set of CFD calculated snapshots), residual minimization, and continuation in the parameter space. In particular, the residual minimization is based on a Broyden-type, quasi-Newton method. Such a method produced spurious solutions in situations in which a GA led to the correct solution. By spurious we mean here solutions that are not close to the CFD solution. The reason was thoroughly checked, concluding that the difficulty is solved by using in the definition of the residual the same governing equations the CFD solver is based upon; namely, including the same artificial dissipation terms. Note that inclusion of the artificial terms is not related with any intrinsic instability of the method, but with an instability that was already present in the CFD solver.

The resulting method is both:

- Quite computationally efficient since calculation of each flow state only requires less than 2 CPU seconds (see Section 4)

\section{3}


while it needs $10 \mathrm{CPU}$ minutes and $6 \mathrm{CPU}$ hours using a ROM based on a GA method and the CFD solver, respectively.

Of course, this computational effort must be added to that associated with the CFD calculation of the snapshots, but such calculation if performed only once. In any event, this is a significant added value envisaging practical engineering design applications. Furthermore, the method's accuracy (which is of the order of 5\% when compared with the CFD results) is comparable to (or even better than) the spread bandwidth $( \pm 10 \%)$ that is typical of experimental testing activities in MEMS.

- Quite robust since the result is fairly independent of the continuation path. Also, the required number of snapshots is reasonably small, and furthermore its location in the parameter space is not critical, even though an optimal selection would allow to further decrease its number. Again, this feature makes the method attractive for engineering design application.

Thus, we expect that the ROM developed in this paper be a step further in current efforts to disseminate the use of reduced order models in industrial environments.

\section{Acknowledgments}

This research has been funded by the Spanish Ministry of Education and Science under Grants DPI2009-07591 (E.B, D.A., and A.V) and TRA2010-18054 (JMV). The authors are indebted with three anonymous referees, whose comments and suggestions helped to clarify the presentation of the results.

\section{Appendix A. The gradient-like method}

The gradient-like method is designed to calculate the PODmode amplitudes (which are considered together in a vector $\mathbf{a}$ ) that minimize the residual. The method is a (quasi-Newton) Broyden's method, which proceeds in the following steps:

1. The initial values of the amplitudes, $\mathbf{a}^{\mathbf{0}}$, and a positive-definite matrix, $\mathbf{M}^{\mathbf{1}}$, are selected. Here, we select $\mathbf{M}^{\mathbf{1}}=\mathbf{I}=$ the unit matrix.

2. The amplitudes vector $a=\left(a_{1}, \ldots, a_{N}\right)$ is updated using the formula $\mathbf{a}^{n+1}=\mathbf{a}^{n}+\alpha^{n+1} \mathbf{M}^{n+1} \mathbf{g}^{n}$, where $\mathbf{g}\left(g_{i}=\partial_{a_{i}} R\right)$ is the gradient vector, and the scalar $\alpha^{n+1}$ is determined upon onedimensional minimization of the residual $R$, which is done using the, Regula falsi method to solve the equation $\mathbf{g}=0$ (i.e., imposing that the gradient along the associated straight line be zero).

3. The matrix $\mathbf{M}^{\mathrm{n}}$ is updated to $\mathbf{M}^{n+1}$, using the BroydenFletcher-Goldfarb-Shanno (BFGS) method [20], which yields

$$
\begin{aligned}
\mathbf{M}^{n+1}= & \mathbf{M}^{n}+\left(I+\frac{\left(\partial_{1}^{n}\right)^{T} \mathbf{M}^{n} \delta_{1}^{n}}{\left(\delta_{0}^{n}\right)^{T} \delta_{1}^{n}}\right) \frac{\left(\delta_{0}^{n}\right)^{T} \delta_{0}^{n}}{\left(\delta_{0}^{n}\right)^{T} \delta_{1}^{n}} \\
& -\frac{\delta_{0}^{n}\left(\delta_{1}^{n}\right)^{T} \mathbf{M}^{n}+\mathbf{M}^{n} \delta_{1}^{n}\left(\delta_{0}^{n}\right)^{T}}{\left(\delta_{0}^{n}\right)^{T} \delta_{1}^{n}}
\end{aligned}
$$

where $\delta_{0}^{n}=\mathbf{a}^{n}-\mathbf{a}^{n-1}$ and $\delta_{1}^{n}=\mathbf{g}^{n}-\mathbf{g}^{n-1}$. Here, the superscript $T$ denotes the transpose.

4. If the $L_{2}$ norm of the vector $\mathbf{g}^{n+1}$ is smaller than some predefined $\varepsilon_{1}$, then iteration ends. Otherwise, the method proceeds back to step 2 .

After trying several quasi-Newton methods, the BFGS formula above has been chosen as the one that exhibits better performances in various $\mathrm{POD} /$ residual minimization problems. The resulting method exhibits a super linear convergence rate, a low time cost (the number of operations scales with the square of the number of retained POD modes), and robustness (the matrix $\mathbf{M}$ is always positive-definite).

\section{Appendix B. The continuation method}

Continuation is performed with a natural parameter continuation [24] method straight lines in the parameter space. It is convenient to scale the parameters such that they vary from 0 to 1 . In the test case considered in this paper, this is made using the rescaled Reynolds number $(\operatorname{Re}-50) / 200$ and the wall temperature $T_{\text {wall. }}$ In each step of the continuation method, the gradient-like method is applied as described in Appendix A. The continuation method starts in a given snapshot and proceeds in four steps:

1. The initial guess of the amplitudes vector is calculated as the projection of selected snapshot on the POD manifold. The initial step size is selected (as, e.g., $d s=0.05$ ).

2. The parameters are updated moving a step of length $d s$ along the continuation line.

3. For the new parameter values, the amplitudes are calculated running the gradient-like method in Appendix A using as initial guess the solution in the former continuation step. Now, we have three possibilities:

i. If convergence does not occur in a number of steps ten times larger than the number of unknowns (the total number of amplitudes in the four flow variables), then $d s$ is halved and the process is repeated from step 2 .

ii. Otherwise, the converged solution is accepted at the current parameter values. Concerning $d s$, (a) it is doubled at the next continuation step if convergence occurs in a number of steps smaller than the number of unknowns and (b) it is maintained otherwise.

4. If the final point in the continuation curve is reached the process ends. Otherwise, the process proceeds back to step 3 with the continuation step $d s$ as defined above.

\section{Appendix C. The genetic algorithm}

The genetic algorithm (GA) is used to obtain the global minimum of the residual defined in Eq. (4), which depends on $m=6 \times n$ variables (the amplitudes $A_{j k}$ ). The algorithm uses $N_{I}$ individuals, each of whom exhibits $m$ chromosomes (the amplitudes $A_{j k}$ ); each chromosome in turn consists of 8 genes, which are the bits that codify this particular amplitude. Fitness of an individual is defined according to the value of the residual (4) associated to their genes. The GA allows the individuals to compete among themselves, mutating, and breeding, as follows. The algorithm uses an initial number, $0.1 \times N_{l}$, of equal individuals with the genes obtained by POD + interpolation, and the remaining $0.9 \times N_{I}$ with randomly selected genes. At the beginning of each generation, the individuals are ordered according to their fitness. The first $0.1 \times N_{I}$ individuals, known as elite individuals, survive to the next generation. The remaining $0.9 \times N_{I}$ individuals compete randomly among themselves as follows: two randomly chosen individuals compare their fitness and the best fit survives; such competition is done $0.9 \times N_{I}$ times. Then, the survivors cross their genes in randomly chosen pairs to produce a maximum of $0.9 \times N_{I}$ new individuals whose genes are chosen randomly from the genes of the parents. Another $0.1 \times N_{I}$ genes of the new individuals (excluding the elite) suffer a further random mutation. The resulting new individuals plus the elite individuals form the 
population for the next generation. The process is stopped if either (a) the fitness of the best fit individual remains unchanged for 50 generations, or (b) $N_{G}$ generations occur without accomplishing condition (a). In either case, the individual with the lowest fitness is considered the optimal individual and its genes are assumed to codify the solution of the minimization problem.

\section{References}

[1] Bui-Thanh, Proper Orthogonal Decomposition Extensions and Their Applications in Steady Aerodynamics, Master Thesis: Singapore-MIT Alliance 2003.

[2] L. Lorente, J.M. Vega, A. Velázquez, Generation of aerodynamic databases using high order singular value decomposition, J. Aircraft 25 (2008) 1779-1788.

[3] D. Amsallem, C. Farhat, An interpolation method for adapting reduced-order models and applications to aeroelasticity, AIAA J. 46 (2008) 1803-1813.

[4] D. Amsallem, J. Cortial, K. Calberg, C. Farhat, A method for interpolating manifolds structural dynamics reduced-order models, Int. J. Numer. Methods Eng. 80 (2009) 1241-1258.

[5] T.R. Smith, J. Moehlis, P. Holmes, Low-dimensional modelling of turbulence using the proper orthogonal decomposition: a tutorial, Nonlinear Dyn. 41 (2005) 275-307.

[6] B. Galletti, C.H. Bruneau, C. Zannetti, A. Iollo, Low order modeling of laminar flow regimes past a confined square cylinder, J. Fluid Mech. 194 (2004) 92-116.

[7] S. Sirisup, G.E. Karnadakis, A spectral viscosity method for correcting the long term behavior of POD models, J. Comp. Phys. 194 (2004) 92-116.

[8] J. Burkhard, M. Gunzburger, H.C. Lee, POD and CVT based reduced order modeling of Navier-Stokes flows, Comp. Meth. Appl. Mech. Eng. 196 (2006) 337-355.

[9] M.F. Barone, I. Kalashnikova, D.J. Segalman, H. Thornquist, Stable Galerkin reduced order models for linearized compressible flow, J. Comput. Phys. 288 (2009) 1932-1946.

[10] I. Kalashnikova, M.F. Barone, On the stability and convergence of a Galerkin reduced order model (ROM) of compressible flow with solid wall and far-field boundary treatment, Int. J. Numer. Methods Eng. 83 (2010) 1345-1375.
[11] M.-L. Rapun, J.M. Vega, Reduced order models based on local pod plus Galerkin projection, J. Comp. Phys. 229 (2010) 3046-3063.

[12] P. LeGresley, J. Alonso, Investigation of Non-Linear Projection for POD Based Reduced Order Models for Aerodynamics (2001) AIAA Paper 20010926.

[13] D. Alonso, A. Velazquez, J.M. Vega, Robust reduced order modeling of heat transfer in a back step flow, Int. J. Heat Mass Transfer 52 (2009) 1149-1157.

[14] Y. Rouizi, Y. Favennec, J. Ventura, D. Petit, Numerical model reduction of 2D steady incompressible laminar flow: application on the flow over a backward facing step, J. Comp. Phys. 228 (2009) 2239-2255.

[15] E. Bache, J.M. Vega, A. Velazquez, Model reduction in fluid-thermal problems with variable geometry, Int. J. Thermal Sci. 49 (2010) 2376-2384.

[16] D. Alonso, A. Velazquez, J.M. Vega, A method to generate computationally efficient reduced order models, Comp. Meth. Appl. Mech. Eng. 198 (2009) 2683-2691.

[17] P. Astrid, S. Weiland, K. Willcox, T. Backx, Missing point estimation methods in models described by proper orthogonal decomposition, IEEE Trans. Autom. Control 53 (2008) 2237-2250.

[18] T. Braconnier, M. Ferrier, J.C. Jouhaud, M. Montagnac, P. Sagaut, Towards and adaptive POD/SVD surrogate model for aeronautic design, Comput. Fluids 40 (2011) 195-209.

[19] L.S. Lorente, J.M. Vega, A. Velazquez, Finding the Smallest Manifold that Contains All the Information Required to Generate a Multiparametric Aerodynamics Database. Aerospace Sci. Tech., submitted for publication.

[20] R. Fletcher, Practical Methods of Optimization. John Wiley \& Sons, 2007.

[21] F.P. Incropera, D.P. Dewitt, Introduction to Heat Transfer. John Wiley \& Sons, 1996, Table A-6, pp. 764

[22] B. Mendez, A. Velazquez, Finite point solver for the simulation of 2-D laminar incompressible unsteady flows, Comp. Meth. Appl. Mech. Eng. 193 (2004) 825-848.

[23] A. Velazquez, J.R. Arias, B. Mendez, Laminar heat transfer enhancement downstream of a backward facing step by using a pulsating flow, Int. J. Heat Mass Transfer 51 (2008) 2075-2089.

[24] H.B. Keller, Lectures on Numerical Methods on Bifurcation Problems. Tata Institute of Fundamental Research, Springer-Verlag, 1986. 